\title{
Eficácia de herbicidas isolados e em mistura para o controle de Amaranthus hybridus
}

\section{Efficacy of individual and mixed herbicides to control}

\author{
Amaranthus hybridus \\ João Henrique Corte Cervoni ${ }^{1}$, Wilson Roberto Cerveira Junior ${ }^{1}$, Adilson Ferreira da Silva ${ }^{1}$, \\ Claudinei da $\mathrm{Cruz}^{2}$ \\ ${ }^{1}$ Faculdade de Ciências Agrárias e Veterinária (FCAV), Universidade Estadual Paulista (UNESP) - \\ Jaboticabal (SP), Brasil. \\ ${ }^{2}$ Centro Universitário da Fundação Educacional de Barretos (UNIFEB) - Barretos (SP), Brasil.
}

\section{Resumo}

O objetivo deste estudo foi avaliar a eficácia dos herbicidas glyphosate e saflufenacil isolados e em misturas para o controle do caruru (Amaranthus hybridus). Para tanto, sementes de A. hybridus foram semeadas em vasos com capacidade para 300,0 g, com uma mistura de solo e substrato orgânico $(2: 1 \mathrm{v} / \mathrm{v})$. A aplicação foi realizada quando as plantas estavam com três pares de folhas verdadeiras, e as doses testadas no primeiro experimento foram: i) $960,0 \mathrm{~g} \mathrm{ha}^{-1}$ glyphosate; ii) $84,0 \mathrm{~g} \mathrm{ha}^{-1}$ saflufenacil; iii) $480,0 \mathrm{~g} \mathrm{ha}^{-1}$ glyphosate $+84,0 \mathrm{~g} \mathrm{ha}^{-1}$ saflufenacil; iv) 480,0 $\mathrm{g} \mathrm{ha}^{-1}$ glyphosate $+42,0 \mathrm{~g} \mathrm{ha}^{-1}$ saflufenacil; v) 480,0 $\mathrm{g} \mathrm{ha}^{-1}$ glyphosate $+28,0 \mathrm{~g} \mathrm{ha}^{-1}$ saflufenacil e vi) controle (testemunha); no segundo foi i) $480 \mathrm{~g} \mathrm{ha}^{-1}$ glyphosate; ii) $112,0 \mathrm{~g} \mathrm{ha}^{-1}$ saflufenacil; iii) $240,0 \mathrm{~g} \mathrm{ha}^{-1}$ glyphosate $+84,0 \mathrm{~g} \mathrm{ha}^{-1}$ saflufenacil; iv) $240,0 \mathrm{~g} \mathrm{ha}^{-1}$ glyphosate + $42,0 \mathrm{~g} \mathrm{ha}^{-1}$ saflufenacil; v) 240,0 $\mathrm{g} \mathrm{ha}^{-1}$ glyphosate $+28,0 \mathrm{~g} \mathrm{ha}^{-1}$ saflufenacil; vi) $140,0 \mathrm{~g} \mathrm{ha}^{-1}$ saflufenacil e vii) controle (testemunha). A avaliação de eficácia foi realizada em 3, 7, 15, 21 e 30 dias após a aplicação (DAA). Para o controle do A. hybridus, o glyphosate em $960,0 \mathrm{~g} \mathrm{ha}^{-1}$ apresentou $100 \%$ de controle a partir de 21 DAA, e em $480 \mathrm{~g} \mathrm{ha}^{-1}$ o controle foi de $80 \%$ em 30 DAA; para o saflufenacil, o controle não foi superior a $70 \% \mathrm{em}$ todas as doses utilizadas, porém todas as misturas dos herbicidas glyphosate e saflufenacil foram superiores a 90\% em 30 dias após a aplicação. Assim, o glyphosate isolado e em mistura com o saflufenacil pode ser utilizado no controle do $A$. hybridus.

Palavras-chave: plantas daninhas; controle químico; manejo.

\begin{abstract}
This study aimed to evaluate the effectiveness of glyphosate and isolated saflufenacil and mixtures to control pigweed (Amaranthus hybridus). To this end, A. hybridus seeds were sown in pots with $300.0 \mathrm{~g}$ capacity with a mixture of soil and organic substrate $(2: 1 ; \mathrm{v} / \mathrm{v})$. The application was done when the plants presented with three pairs of true leaves and the doses tested in the first experiment were: i) $960.0 \mathrm{~g} \mathrm{ha}^{-1}$ glyphosate; ii) $84.0 \mathrm{~g} \mathrm{ha}^{-1}$ saflufenacil; iii) $480.0 \mathrm{~g} \mathrm{ha}^{-1}$ glyphosate $+84.0 \mathrm{~g} \mathrm{ha}^{-1}$ saflufenacil; iv) $480.0 \mathrm{~g} \mathrm{ha}^{-1}$ glyphosate $+42.0 \mathrm{~g} \mathrm{ha}^{-1}$ saflufenacil; v) $480.0 \mathrm{~g} \mathrm{ha}^{-1}$ glyphosate $+28.0 \mathrm{~g} \mathrm{ha}^{-1}$ saflufenacil and vi) control (control). The second experiment used i) $480 \mathrm{~g} \mathrm{ha}^{-1}$ glyphosate; ii) $112.0 \mathrm{~g} \mathrm{ha}^{-1}$ saflufenacil; iii) $240.0 \mathrm{~g}$ $\mathrm{ha}^{-1}$ glyphosate $+84.0 \mathrm{~g} \mathrm{ha}^{-1}$ saflufenacil; iv) $240.0 \mathrm{~g} \mathrm{ha}^{-1}$ glyphosate $+42.0 \mathrm{~g} \mathrm{ha}^{-1}$ saflufenacil; v) 240.0
\end{abstract}

Autor para correspondência: João Henrique Corte Cervoni - Faculdade de Ciências Agrárias e Veterinária (FCAV), Universidade Estadual Paulista (UNESP) - Campus Jaboticabal, Via de acesso Prof. Donato Castellane, s/n - CEP: 14884-900 - Jaboticabal (SP), Brasil - E-mail: joaocervoni@hotmail.com

Recebido em: 08 de novembro de 2016

Aceito para publicação em: 09 de julho de 2017 
$\mathrm{g} \mathrm{ha}^{-1}$ glyphosate $+28.0 \mathrm{~g} \mathrm{ha}^{-1}$ saflufenacil; vi) $140.0 \mathrm{~g} \mathrm{ha}^{-1}$ saflufenacil, and vii) control. The efficacy evaluation was performed at 3, 7, 15, 21 and 30 days after application (DAA). For the analysis of $A$. hybridus control, glyphosate in $960.0 \mathrm{~g} \mathrm{ha}^{-1}$ showed $100 \%$ control from 21 DAA and $480 \mathrm{~g} \mathrm{ha}^{-1}$ revealed $80 \%$ control at 30 DAA. Saflufenacil showed control of no more than $70 \%$ in all doses tested, but any mixture of glyphosate; and saflufenacil was greater than $90 \%$ at 30 days after application. Thus, glyphosate alone and mixed with saflufenacil can be used in the control of $A$. hybridus.

Keywords: weeds; chemical control; management.

\section{Introdução}

As plantas daninhas ocorrem espontaneamente em áreas de interesse humano, caracterizadas por seu elevado poder de disseminação e podem causar grandes perdas às principais culturas de interesse econômico (SILVA et al., 2008).

O controle de plantas daninhas pode ser realizado pelo método físico, que consiste na utilização de ferramentas ou implementos agrícolas; o biológico, com a utilização de inimigos naturais, como insetos, fungos entre outros; e o químico, que é o uso de produtos, basicamente herbicidas, que intoxicam as plantas (SIQUEIRA et al., 2014) e são indispensáveis para o controle de plantas daninhas, pela facilidade, eficácia, custo do tratamento, rápido resultado e redução do tráfego pesado em áreas cultivadas (OLIVEIRA JUNIOR et al., 2011).

Dentre os herbicidas empregados, o glyphosate se destaca por ser não seletivo e de ação sistêmica, pertencente ao grupo químico das glicinas, que atua na inibição da enzima 5-enolpiruvil-chiquimato3-fosfatosintase (EPSPS). Este herbicida é muito utilizado no controle de plantas daninhas, em pós-emergência, como Commelina benghalensis (CORREIA et al., 2008; CARVALHO et al., 2015) e na dessecação de plantas para formação de cobertura, como Urochloa decumbens, $U$. brizantha e $U$. ruziziensis (TIMOSSI et al., 2006; SILVA et al., 2013).

O saflufenacil é outro herbicida com potencial, que foi desenvolvido para controle das plantas daninhas em pré-emergência, pré-emergência incorporado e pós-emergência, pertence à família das Pirimidinedione, atua na inibição da enzima protoporfirinogênio oxidase (PROTOX) (GROSSMANN et al., 2011; OLIVEIRA JUNIOR et al., 2011).

Dentre as principais famílias de plantas daninhas, Amaranthaceae possui uma ampla variedade de espécies que apresentam suscetibilidade variável aos herbicidas (CARVALHO et al., 2006;
RAIMONDI et al., 2010). Como o clomazone, que promoveu excelente controle de A. viridis, o Diuron, pendimentalin e S-metolachlor com excelente controle para $A$. hybridus, A. lividus, A. spinosus e $A$. viridis (RAIMONDI et al., 2010), o herbicida fomesafen também foi eficaz no controle de A. hybridus (ZANATTA et al., 2008).

Estas espécies são consideradas como problemas para a agricultura, pois têm uma alta competitividade com as culturas por água, luz, espaço e nutrientes (SILVA et al., 2010; BARROSO et al., 2012). Dentre as plantas daninhas que causam prejuízos econômicos está A. hybridus, que pertence à família Amaranthaceae, conhecida como caruru ou caruru-roxo, uma planta daninha invasora que ocorre em todos os estados brasileiros (MARCHIORETTO et al., 2010). Possui hábito de crescimento vertical ereto com ramificações primárias (CARVALHO et al., 2008). O caruru pode causar grandes interferências nas culturas como: redução inicial da área foliar e na taxa de crescimento, redução considerável da produtividade, qualidade da colheita e danos às colhedoras (SMITH et al., 2000; BERRY et al., 2006; MANABE et al., 2014).

A utilização incorreta dos herbicidas, com o uso repetitivo do mesmo modo de ação, elevadas doses e longo período residual podem proporcionar aumento de biótipos tolerantes e, com passar do tempo, o surgimento da resistência (CARVALHO et al., 2006). Existem vários relatos de caruru resistente no mundo e são citados por diversos trabalhos como: Ryan (1970) (Amaranthus hybridus); Christoffoleti et al. (1994) (Amaranthus hybridus e Amaranthus retroflexus) Lovell et al. (1996) e Foes et al. (1998) (Amaranthus rudis); Kissmann e Groth (1999) (Amaranthus palmeri) e Carvalho et al. (2006) (Amaranthus deflexus). No Brasil há dois casos de caruru resistente, o A. retroflexus e A. viridis, ambos resistentes aos herbicidas inibidores de FSII (atrazine) e inibidores 
da ALS (prometryn e trifloxysulfuron-sodium) (FRANCISCHINI, 2011).

Assim, a utilização de mistura de herbicidas pode ser uma forma de ampliar o espectro de controle de plantas daninhas, reduzir os custos de produção, as doses aplicadas no campo e as chances de surgimento de biótipos resistentes. Para tanto, o objetivo deste estudo foi avaliar a eficácia de controle dos herbicidas glyphosate e saflufenacil isolados e em misturas para o controle do caruru (Amaranthus hybridus).

\section{Material e Métodos}

O caruru (Amaranthus hybridus) foi semeado em vasos com capacidade de 300,0 gramas contendo uma mistura de solo e substrato comercial $\operatorname{Plantmax}^{\circledR}(2: 1 \mathrm{v} / \mathrm{v})$, em casa de vegetação. Após a emergência das plantas foi realizado o desbaste, deixando quatro plantas vigorosas em cada vaso. Após atingirem $10 \mathrm{~cm}$ de altura com emissão de três pares de folhas verdadeiras foi realizada a aplicação dos herbicidas.

Os herbicidas utilizados foram o glyphosate (480 g L $^{-1}$ ), na formulação Roundup Ready ${ }^{\circledR}$, e o Saflufenacil $\left(700 \mathrm{~g} \mathrm{~kg}^{-1}\right)$, na formulação Heat ${ }^{\circledR}$.

Para aplicação dos herbicidas foi usado um pulverizador manual pressurizado por $\mathrm{CO}_{2}$, com pressão constante de 2,0 bar, equipado com barra munida de quatro pontas do tipo XR 110.02, com espaçamento entre pontas e altura da planta daninha de $0,5 \mathrm{~m}$ e velocidade de aplicação de $1 \mathrm{~m} / \mathrm{s}$, que corresponde a um volume de calda de 200,0 L ha' $\mathrm{L}^{-1}$. As condições climáticas foram: vento entre 3,0 e $3,5 \mathrm{~km} \mathrm{~h}^{-1}$; umidade relativa de 63 e $67 \%$ e temperatura de $27,0{ }^{\circ} \mathrm{C}$.

No primeiro experimento foram utilizados os seguintes tratamentos: i) glyphosate ( $\left.960 \mathrm{~g} \mathrm{ha}^{-1}\right)$; ii) saflufenacil ( $\left.84 \mathrm{~g} \mathrm{ha}^{-1}\right)$; iii) glyphosate + saflufenacil (480 $\left.\mathrm{g} \mathrm{ha}^{-1}+84 \mathrm{~g} \mathrm{ha}^{-1}\right)$; iv) glyphosate + saflufenacil $\left(480 \mathrm{~g} \mathrm{ha}^{-1}+42 \mathrm{~g} \mathrm{ha}^{-1}\right)$; v) glyphosate + saflufenacil $\left(480 \mathrm{~g} \mathrm{ha}^{-1}+28 \mathrm{~g} \mathrm{ha}^{-1}\right) \mathrm{e}$ vi) controle (testemunha), com 5 cinco réplicas por tratamento.

No segundo experimento foram utilizados os tratamentos: i) glyphosate (480 $\left.\mathrm{g} \mathrm{ha}^{-1}\right)$; ii) saflufenacil $\left(112,0 \mathrm{~g} \mathrm{ha}^{-1}\right)$; iii) glyphosate + saflufenacil (240 $\left.\mathrm{g} \mathrm{ha}^{-1}+84 \mathrm{~g} \mathrm{ha}^{-1}\right)$; iv) glyphosate + saflufenacil $\left.\left(240 \mathrm{~g} \mathrm{ha}^{-1}+42 \mathrm{~g} \mathrm{ha}^{-1}\right) ; \mathrm{v}\right)$ glyphosate + saflufenacil $\left(240 \mathrm{~g} \mathrm{ha}^{-1}+28 \mathrm{~g} \mathrm{ha}^{-1}\right)$; vi) saflufenacil (140 $\mathrm{g} \mathrm{ha}^{-1}$ ) e vii) controle (testemunha), também com 5 cinco réplicas por tratamento.
A avaliação da porcentagem de mortalidade das plantas foi realizada em 3, 7, 15, 21, 30 dias após a aplicação (DAA) e foram atribuídas notas de controle segundo escala de notas (A a E), em que A corresponde de 91 a $100 \%$ de controle; B 71 a $90 \%$; C 61 a 70\%, D 41 a $60 \%$ e E 0 a $40 \%$, de acordo com Asociación Latinoamericana de Malezas (1974) e Sociedade Brasileira da Ciência das Plantas Daninhas (1995).

\section{Resultados e Discussão}

$\mathrm{Na}$ fase inicial do primeiro experimento ocorreu diferença de resposta com a utilização da mistura dos herbicidas glyphosate e saflufenacil. Após três dias da aplicação, $A$. hybridus apresentou controle de 65 a $75 \%$ na utilização da mistura entre glyphosate e saflufenacil. Aos 21 dias, as misturas de glyphosate e saflufenacil $\left(480 \mathrm{~g} \mathrm{ha}^{-1}+84 \mathrm{~g} \mathrm{ha}^{-1}\right.$ e $\left.480 \mathrm{~g} \mathrm{ha}^{-1}+42 \mathrm{~g} \mathrm{ha}^{-1}\right)$ promoveram excelente controle com $100 \%$ de eficácia (Tabela 1).

$\mathrm{O}$ glyphosate isolado na dose de $960 \mathrm{~g} \mathrm{~h}^{-1}$ promoveu eficácia de $60 \%$ em 3 DAA e de $100 \%$ no controle, em 21 DAA. O saflufenacil na dose de $84 \mathrm{~g} \mathrm{ha}^{-1}$ apresentou eficácia de controle de $20 \%$ durante todo o período experimental (Tabela 1).

Os resultados de eficácia de controle obtidos neste estudo foram similares a mesotrione $\left(120,0 \mathrm{~g} \mathrm{ha}^{-1}\right)$ e ametrina (2000,0 $\left.\mathrm{g} \mathrm{ha}^{-1}\right)$, isolados e em mistura $\left(120,0+2000,0 \mathrm{~g} \mathrm{ha}^{-1}\right.$, respectivamente $)$ e entre o mesotrione e metribuzin $\left(120,0+1920,0 \mathrm{~g} \mathrm{ha}^{-1}\right)$ com $100 \%$ de controle para o A. deflexus na cultura da cana-de-açúcar (CARVALHO et al., 2010). Para Zanatta et al. (2008), ocorreu excelente controle do $A$. hybridus com a utilização de fomesafen na dose de $375,0 \mathrm{~g} \mathrm{ha}^{-1}$ aos 22 DAA.

Para Nicolai et al. (2010), ocorreu excelente controle de $A$. viridis pelo herbicida S-metolachlor na dose 2400,0 $\mathrm{g} \mathrm{ha}^{-1}\left(90,0 \%\right.$ ) e ametrina, 2000,0 $\mathrm{g} \mathrm{ha}^{-1}$ $(82,5 \%)$. A mistura de S-metolachlor e ametrina $\left(2880,0+1500,0 \mathrm{~g} \mathrm{ha}^{-1}\right)$ promoveu $93,5 \% \mathrm{de}$ controle, e S-metolachlor, diuron e hexazinone $\left(1920,0+198,0+702,0 \mathrm{~g} \mathrm{ha}^{-1}\right)$ obteve um controle de $98,3 \%$, no período de 90 DAA.

No segundo experimento, o controle foi inexistente com o glyphosate na dose de $480,0 \mathrm{~g} \mathrm{ha}^{-1}$ para o $A$. hybridus no início do experimento (3 e 7 DAA), porém aos 45 DAA a eficácia foi de $90 \%$. O saflufenacil na dose $112,0 \mathrm{~g} \mathrm{ha}^{-1}$ promoveu $85 \%$ de controle aos 45 DAA, porém, em 140,0 $\mathrm{g} \mathrm{ha}^{-1}$ apresentou resultados moderados, com eficácia de $41 \%$ aos 30 DAA (Tabela 2). 
Tabela 1. Notas e eficácia de controle (\%) dos herbicidas glyphosate e saflufenacil isolados e em mistura para A. hybridus ( $1^{\circ}$ experimento).

\begin{tabular}{llllll}
\hline \multirow{2}{*}{ Tratamentos } & \multicolumn{5}{c}{ Dias Após Aplicação (DAA) } \\
\cline { 2 - 6 } & $\mathbf{3}$ & $\mathbf{7}$ & $\mathbf{1 5}$ & $\mathbf{2 1}$ & $\mathbf{3 0}$ \\
\hline Glyphosate $960 \mathrm{~g} \mathrm{ha}^{-1}$ & $\mathrm{C}-60$ & $\mathrm{~B}-80$ & $\mathrm{~B}-95$ & $\mathrm{~A}-100$ & $\mathrm{~A}-100$ \\
Saflufenacil $84,0 \mathrm{~g} \mathrm{ha}^{-1}$ & $\mathrm{E}-10$ & $\mathrm{E}-20$ & $\mathrm{E}-20$ & $\mathrm{E}-20$ & $\mathrm{E}-20$ \\
Glyphosate 480 $\mathrm{g} \mathrm{ha}^{-1}+84,0 \mathrm{~g} \mathrm{ha}^{-1}$ Saflufenacil & $\mathrm{C}-65$ & $\mathrm{~B}-80$ & $\mathrm{~B}-90$ & $\mathrm{~A}-100$ & $\mathrm{~A}-100$ \\
Glyphosate 480 $\mathrm{g} \mathrm{ha}^{-1}+42,0 \mathrm{~g} \mathrm{ha}^{-1}$ Saflufenacil & $\mathrm{C}-65$ & $\mathrm{~B}-80$ & $\mathrm{~B}-80$ & $\mathrm{~A}-100$ & $\mathrm{~A}-100$ \\
Glyphosate 480 $\mathrm{g} \mathrm{ha}^{-1}+28,0 \mathrm{~g} \mathrm{ha}^{-1}$ Saflufenacil & $\mathrm{B}-75$ & $\mathrm{~B}-85$ & $\mathrm{~B}-90$ & $\mathrm{~A}-95$ & $\mathrm{~A}-100$ \\
Controle & $\mathrm{E}-0$ & $\mathrm{E}-0$ & $\mathrm{E}-0$ & $\mathrm{E}-0$ & $\mathrm{E}-0$ \\
\hline
\end{tabular}

Tabela 2. Notas e eficácia de controle (\%) dos herbicidas glyphosate e saflufenacil isolados e em mistura para A. hybridus ( $2^{\circ}$ experimento).

\begin{tabular}{lllllll}
\hline \multirow{2}{*}{ Tratamentos } & \multicolumn{5}{c}{ Dias Após Aplicação (DAA) } \\
\cline { 2 - 7 } & $\mathbf{3}$ & $\mathbf{7}$ & $\mathbf{1 5}$ & $\mathbf{2 1}$ & $\mathbf{3 0}$ & $\mathbf{4 5}$ \\
\hline Glyphosate 480,0 $\mathrm{g} \mathrm{ha}^{-1}$ & E-0 & E-0 & D-50 & B-71 & B-80 & B-90 \\
Saflufenacil 112,0 $\mathrm{g} \mathrm{ha}^{-1}$ & E-0 & E-5 & E-20 & C-61 & C-70 & B-85 \\
Glyphosate 240,0 $\mathrm{g} \mathrm{ha}^{-1}+84,0 \mathrm{~g} \mathrm{ha}^{-1}$ Saflufenacil & E-20 & E-25 & C-65 & B-90 & A-95 & A-100 \\
Glyphosate 240,0 $\mathrm{g} \mathrm{ha}^{-1}+42,0 \mathrm{~g} \mathrm{ha}^{-1}$ Saflufenacil & E-20 & E-25 & C-65 & A-95 & A-95* & A-100 \\
Glyphosate 240,0 $\mathrm{g} \mathrm{ha}^{-1}+28,0 \mathrm{~g} \mathrm{ha}^{-1}$ Saflufenacil & E-10 & E-10 & C-65 & B-90* & B-90 & A-100 \\
Saflufenacil 140,0 g ha $^{-1}$ & E-0 & E-5 & E-10 & E-20 & D-41 & D-41 \\
Controle & E-0 & E-0 & E-0 & E-0 & E-0 & E-0 \\
\hline
\end{tabular}

Nas misturas dos herbicidas glyphosate e salfufenacil, as porcentagens de controle não apresentaram grandes variações na maioria das avaliações (3, 7, 21 e 45 DAA), e foram similares em 15 e 45 DAA, sendo a partir de 21 DAA controle de 90 a $100 \%$ (Tabela 2).

Resultados similares foram obtidos para I. grandifolia aplicadas, em estádio de 1 a 3 pares de folha, com a utilização das misturas entre: cloransulam-methyl + glyphosate $\left(30,2+960,0\right.$ g i.a ha $\left.{ }^{-1}\right)$, chlorimuron-ethyl + glyphosate $\left(12,50+960,0 \mathrm{~g}\right.$ i.a ha $\left.{ }^{-1}\right)$ e imazethapyr + glyphosate $\left(80,0+960,0\right.$ g i.a ha $\left.{ }^{-1}\right)$ com controle de $98,75 \%$, e glyphosate isolado na dose de 960,0 g i.a ha- ${ }^{-1}$ com controle de $99 \%$ em 35 DAA (RAMIRES et al., 2010).

Para Vargas et al. (2007), em Conyza bonariensis resistentes, o controle foi de $95 \%$ com o herbicida 2,4-D $\left(1.005,0 \mathrm{~g} \mathrm{ha}^{-1}\right), 95 \%$ com paraquat $\left(400,0 \mathrm{~g} \mathrm{ha}^{-1}\right)$, $70 \%$ com chlorimuron-ethyl $\left(40,0 \mathrm{~g} \mathrm{ha}^{-1}\right), 80 \%$ com metsulfuron-methyl $\left(4,0 \mathrm{~g} \mathrm{ha}^{-1}\right)$, e com mistura entre diuron + paraquat $\left(200,0+400,0 \mathrm{~g} \mathrm{ha}^{-1}\right) \mathrm{o}$ controle foi de $100 \%$ em 7 DAA, sendo similar aos resultados obtidos no controle $A$. hybridus com as misturas de glyphosate e saflufenacil.

$\mathrm{O}$ intenso uso de produtos à base de glyphosate para controle de plantas daninhas, pode ser a causa para o surgimento de biótipos com resistência para esta molécula. Para tanto, as utilizações da mistura entre as duas moléculas de modo de ação diferentes melhoraram a resposta de eficácia do saflufenacil e permitiu a diminuição da dose do glyphosate aplicado, um ponto importante tanto na preservação do modo de ação ESPS quanto do ponto de vista ambiental. A mistura 240,0 $\mathrm{g} \mathrm{ha}^{-1}$ de glyphosate $+28,0 \mathrm{~g} \mathrm{ha}^{-1}$ do saflufenacil foi a que obteve melhores resultados, pois no decorrer do experimento a eficácia de controle aumentou gradativamente e foi possível obter um controle excelente para o A hybridus.

\section{Conclusão}

Para o controle do A. hybridus, o glyphosate na dose $960,0 \mathrm{~g} \mathrm{ha}^{-1}$ apresentou $100 \%$ de controle a partir de 21 DAA para o saflufenacil o controle não foi superior a $70 \%$, porém a mistura dos herbicidas glyphosate e saflufenacil foi superior a $90 \%$. Assim, o glyphosate isolado e em mistura com o saflufenacil pode ser utilizado no controle do A. hybridus.

\section{Referências}

ASOCIACIÓN LATINOAMERICANA DE MALEZAS - ALAM. Recomendaciones sobre 
unificación de los sistemas de evaluación en ensayos de control de malezas. $A L A M$, v. 1, n. 1 , p. 35-38, 1974.

BARROSO, A. A. M. et al. Efeito da densidade e da distancia de caruru-de-mancha e amendoinbravo na cultura do feijoeiro. Planta Daninha, v. 30, n. 1, p. 47-53, 2012.

BERRY, A. D. et al. Smooth pigweed (Amaranthus hybridus L.) and livid amaranth (Amaranthus lividus) interference with cucumber (Cucumis sativus). Weed Technology, v. 20, n. 1, p. 227-231, 2006.

CARVAlHO, F. T. D. et al. Controle de dez espécies daninhas em cana-de-açúcar com o herbicida mesotrione em mistura com ametryn e metribuzin. Planta Daninha, v. 28, n. 3, p. 585590, 2010.

CARVALHO, F. P. et al. Controle de trapoeraba com diferentes formulações de glyphosate. Revista Brasileira de Herbicidas, v. 14, n. 3, p. 194-199, 2015.

CARVALHO, S. D.; LÓPEZ-OVEJERO, R. F.; CHRISTOFFOLETI, P. J. Crescimento e desenvolvimento de cinco espécies de plantas daninhas do gênero Amaranthus. Bragantia, v. 67, n. 2, p. 317-326, 2008.

CARVALHO, S. J. P. et al. Suscetibilidade diferencial de plantas daninhas do gênero Amaranthus aos herbicidas trifloxysulfuron-sodium e chlorimuronethyl. Planta Daninha, v. 24, n. 3, p. 541-548, 2006.

CHRISTOFFOLETI, P. J.; VICTÓRIA FILHO, R.; SILVA, C. D. Resistência de plantas daninhas aos herbicidas. Planta Daninha, v. 12, n. 1, p. 13-20, 1994.

CORREIA, N. M.; DURIGAN, J. C.; LEITE, G. J. Seletividade da soja transgênica tolerante ao glyphosate e eficácia de controle de Commelina benghalensis com herbicidas aplicados isolados e em misturas. Bragantia, v. 67, n. 3, p. 663-671, 2008.

FOES, M. J. et al. A biotype of common waterhemp (Amaranthus rudis) resistant to triazine and ALS herbicides. Weed Science, v. 46, n. 5, p. 514-520, 1998.
FRANCISCHINI, A. C. Identificação de resistência ais herbicidas utilizados no algodoeiro em biótipos de Amaranthus retroflexus e Amaranthus viridis oriundos das regiões produtoras da Bahia, Goiás, Mato Grosso e Mato Grosso do Sul (Mestrado em Agronomia) - Universidade Estadual de Maringá, Maringá, 2011.

GROSSMANN, K. et al. Saflufenacil: biokinetic properties and mechanism of selectivity of a new protoporphyrinogen IX oxidade inhibiting herbicide. Weed Science, v. 59, n. 3, p. 290-298, 2011.

KISSMANN, K. G.; GROTH, D. Plantas infestantes e nocivas. São Paulo: BASF, 1999. 978 p.

LOVELL, S. T. et al. Imidazolinone and sulfonylurea resistance in a biotype of common waterhemp (Amaranthus rudis). Weed Science, v. 44, n. 4, p. 789-794, 1996.

MANABE, P. M. S. et al. Efeito da competição de plantas daninhas na cultura do feijoeiro. Bioscience Journal, v. 31, n. 2, p. 333-343, 2014.

MARCHIORETTO, M. S.; SENNA, L. R.; SIQUEIRA, J. Amaranthaceae. In: FORZZA, R. C. et al. Catálogo de plantas e fungos do Brasil. Rio de Janeiro: Jardim Botânico do Rio de Janeiro, 2010.

NICOLAI, M. et al. Programas de manejo químico de plantas daninhas em plantio de canade-açúcar fundamentados em duas aplicações de herbicidas. Revista Agrogeoambiental, v. 2, n. 3 , p. 135-144, 2010.

OLIVEIRA JUNIOR, R. S.; CONSTANTIN, J.; INOUE, M. H. Biologia e manejo de plantas daninhas. Omnipax, v. 8, p. 193, 2011.

RAIMONDI, M. A. et al. Atividade residual de herbicidas aplicados ao solo em relação ao controle de quatro espécies de Amaranthus. Planta Daninha, v. 28, n., 4, p. 1073-1085, 2010.

RAMIRES, A. C. et al. controle de Euphorbia heterophylla e Ipomoea grandifolia com a utilização de glyphosate isolado ou em associação com latifolicidas. Planta Daninha, v. 28, n. 3, p. 621-629, 2010. 
RYAN, G. F. Resistance of common groundsel to simazine and atrazine. Weed Science, v. 18, n. 5, p. 614-616, 1970.

SILVA, A. M. A.; DANTAS, I. C.; MEDEIROS, P. R. Levantamento florístico das plantas daninhas em um parque público de Campina Grande, Paraíba, Brasil. Biotemas, v. 21, n. 4, p. 7-14, 2008.

SILVA, B. P. et al. Interferência de caruru-demancha, maria-pretinha, picão-preto e tiririca em tomateiro industrial. Bragantia, v. 69, n. 2, p. 313-318, 2010.

SILVA, U. R. et al. Eficácia do glyphosate na dessecação de espécies de Urochloa. Revista Brasileira de Herbicidas, v. 12, n. 2, p. 202-209, 2013.

SIQUEIRA, R. H. S. et al. agregação de um latossolo vermelho-amarelo submetido a métodos de controle de plantas invasoras na cultura do café. Revista Brasileira de Ciência do Solo, v. 38, n. 4, p. 1128-1134, 2014.
SMITH, D. T.; BAKER, R. V.; STEELE, G. L. Palmer amaranth (Amaranthus palmeri) impacts on yield, harvesting and ginning in dryland cotton (Gossypium hirsutum). Weed Technology, v. 14, n. 1, p. 122-126, 2000.

SOCIEDADE BRASILEIRA DA CIÊNCIA DAS PLANTAS DANINHAS - SBCPD. Procedimentos para instalação, avaliação e análise de experimentos com herbicidas. Londrina: SBCPD, 1995. v. 42.

TIMOSSI, P. C.; DURIGAN, J. C.; LEITE, G. J. Eficácia de glyphosate em plantas de cobertura. Planta Daninha, v. 24, n. 3, p. 475-480, 2006.

VARGAS, L. et al. Buva (Conyza bonariensis) resistente ao glyphosate na região sul do Brasil. Planta Daninha, v. 25, n. 3, p. 573-578, 2007.

ZANATTA, J. F. et al. Teores de água no solo e eficácia do herbicida fomesafen no controle de Amaranthus hybridus. Planta Daninha, v. 26, n. 1, p. 143-155, 2008. 